\title{
Infrared Observations of Peculiar Carbon Stars
}

\author{
ANA ULLA ${ }^{1,2}$, PETER THEJLL ${ }^{3}$, TÔNU KIPPER ${ }^{4}$, \\ and UFFE GRÅE JØRGENSEN ${ }^{5}$ \\ ${ }^{1}$ Instituto Astronomico de las Canarias, Tenerife, Spain \\ ${ }^{2}$ Laboratorio de Astrofísica Espacial y Física Fundamental \\ Madrid, Spain \\ ${ }^{3}$ NORDITA, Copenhagen, Denmark \\ 4 Tartu Astrophysical Observartory, Toravere, Estonia \\ ${ }^{5}$ Niels Bohr Institute, Copenhagen, Denmark
}

We present a uniform and high-quality set of infrared photometric $(J H K)$ observations of the 6 peculiar carbon giant stars V Ari, UV Cam, BD +34 911 , TU Gem, $\mathrm{BD}+57^{\circ} 2161$ and $\mathrm{BD}+34^{\circ} 4134$. Comparison of the $J-H$ and $H-K$ colors to other $\mathrm{C}$ stars indicates that our sample of stars has smaller color indices and this in turn implies that they are hotter if we use the standard assumption that there is a link between these colors and the temperatures. Furthermore, using standard assumptions we derive estimates of their effective temperatures, gravities, luminosities and distances.

This paper has appeared in $A \& A 319,244,1997$. 